\title{
Gazes induce similar sequential effects as arrows in a target discrimination task
}

\author{
Qian Qian ${ }^{1}$, Xiaoting Wang ${ }^{1}$, Miao Song ${ }^{2}$, and Feng Wang ${ }^{1}$ \\ ${ }^{1}$ Yunnan Key Laboratory of Computer Technology Applications, Kunming University of Sci- \\ ence and Technology, 727 Jing Ming Nan Lu, Kunming, Yunnan, 650500, China \\ qianqian yn@126.com \\ ${ }^{2}$ Information Engineering College, Shanghai Maritime University, Shanghai, 200135, China
}

\begin{abstract}
Symbolic cueing paradigm has been widely used to investigate the attention orienting induced by centrally-presented gaze or arrow cues. Previous studies have found a sequence effect in this paradigm when arrows are used as central cues and simple detection tasks are included. The present study investigated the universality of the sequence effect with gaze cues and in discrimination tasks. It was found that sequence effects are not limited to specific cue types or specific tasks, and the sequence effect can even generalize across different cue types (from gaze to arrow, or from arrow to gaze). In addition, the sequence effect is not influenced by the repetition and switch of target identities (along with response keys). The results suggest that sequential processing is a common mechanism in attention orienting systems, and support the automatic retrieval hypothesis more than the strategy adjustment account.
\end{abstract}

Keywords: Attention orienting. Cueing effect. Sequence effect. Memory retrieval.

\section{Introduction}

Orienting of attention refers to the ability for our attention system to be able to select pertinent input for further processing according to external cues [1]. It has been found that perception of a pointing arrow is enough to shift our attention to the pointed location reflexively [2]. Besides arrows, another people's gaze can also shift attention even when these symbolic cues are uninformative for our task in hand [3]. In a typical study of the symbolic cueing, observers were presented with a centrally-presented symbolic cue (e.g., a gaze or an arrow) indicating left or right, and after a certain time interval (stimulus onset asynchrony (SOA)) were instructed to respond to the appearance of a target to the left or right of the central cue. Although observers were told that the direction of the central cue did not predict where the target would occur, reaction time (RT) was reliably faster when the cue direction was toward (i.e., valid trials), rather than away from (i.e., invalid trials), the target. This facilitation of RT between valid and invalid trials is referred to as cueing effect, which is considered to be evidence of attention orienting. 
Several recent studies [4,5] reported a sequence effect in symbolic cueing paradigm. That is, when using a predictive central arrow cue (i.e., the ratio of valid trials among all trials were $80 \%$ ), the cueing effect in one trial is significant smaller when the previous trial is an invalid trial than when it is a valid trial. Since the cues of these studies successfully predict the target locations in most of the trials, the sequence effect has been attributed to the continuous updating of the predictive value that participants assign to the spatial cue. In other words, participants adapt their utilization of the cue depending on whether it is correctly or wrongly directed their attention on the previous trial. On the contrary, another explanation about the sequence effect observed in these studies is automatic memory processes in which information of previous trials is automatically retrieved from memory to facilitate performance on current trials. For example, when the previous trial type (valid or invalid) is consistent with the current trial type, performance will be facilitated, whereas when the previous and current trial types differ, performance is slowed due to the conflict between the two trial types. This automatic retrieval hypothesis is in line with the view from peripheral cueing studies [6] and further supported by the results of [7], in which the sequence effect was still found when the arrow cues did not predict the target location and the participants were explicitly asked to ignore the arrow cues.

In symbolic cueing studies, arrow and gaze cues are two representative attentional cues. However, so far as we know, the sequence effect of symbolic cueing is investigated only with arrow cues and only in a simple detection task [4], [7]. Therefore, there still have some questions for the sequence effect. First, whether sequence effects can be found in other cueing tasks. For example, a simple detection task only need participants to press one key whenever they detect the targets, but a discrimination task will ask participants to choose response keys according to the target identities. In the simple detection task, there are only one target and one response , but in the discrimination task, target identities and responses either repeated or switched between consecutive trials. We still don't know whether sequence effects exist in a discrimination task, and whether the change of target identities and responses between trials influences the sequence effect; Second, whether or not gaze cues induce sequence effects, this question is important because it can help to answer the question whether or not sequential processes in human attention orienting systems are common mechanisms responding to central symbolic cues; Third, if sequential processes are common mechanisms, whether or not the sequence processes of symbolic cueing can generalize across different cue types (e.g., from gaze to arrow cues), and whether there are differences between them.

In experiment 1 , sequence effects were tested with a central gaze cue. If sequence effects are common mechanisms in symbolic cueing, significant sequence effects should also be observed in gaze cue condition. In addition, different from previous studies, a discrimination task, instead of a simple detection task, was used to test the flexibility of the sequence effect. If sequence effects are robust phenomenon in symbolic cueing, significant sequence effects should also be observed with a discrimination task. Considering that in the previous study [7], significant sequence effects were only found when the SOA of previous trials was relatively long, we expect that sequence effects of gaze cueing are significant, but only for long SOA conditions. 
Another advantage for choosing a discrimination task instead of a simple detection task is that there are two kinds of targets, and participants need to press different buttons to respond to the different targets. Therefore, target identities and its corresponding responses will either repeated or switched between consecutive trials. By using a simple detection task, previous study [7] found a significant influence of target location alternation on sequence effects in arrow cueing. However, because participants only need to press one specific button to respond to the appearance of one specific target in a simple detection task, whether the sequence effect is also influenced by the repetition and switch of target identities (along with its corresponding responses) is still unknown. With a discrimination task, we can investigate how target locations and target identities affect sequence effects.

Experiment 2 was aimed to compare the sequence effects induced by gaze and arrow cues. During the experimental trials, gaze and arrow cues were randomly mixed to form four kinds of cue sequence conditions between consecutive trials: gaze-gaze sequence, gaze-arrow sequence, arrow-gaze sequence, and arrow-arrow sequence. This experimental design had several advantages. First, the sequence effects induced by gaze and arrow cues could be compared directly by comparing the magnitude of sequence effects between gaze-gaze sequence and arrow-arrow sequence conditions. Different from arrow cues, cueing effects by gazes are based on mechanisms specialized for gaze perception [8]. The biological-significance of the gaze cues should make it more meaningful and important for participants. Therefore, according to strategy adjustment account, participants may adapt their utilization of the gaze cues more strongly than arrow cues, leading to an enhanced sequence effect in gaze cueing. However, an automatic retrieval hypothesis will predict similar sequence effects for both gaze and arrow cues.

Second, the experimental design allowed us to investigate whether the sequence effect could generalize across cue types. According to the strategy adjustment account, sequence effects are originated from continuous updating of the predictive value that participants assign to the cue. Therefore, the change of cue types between consecutive trials should eliminate the sequential processing, because the last cue is new for participants and there is no reason for participants to update the predictive value of a new cue. On the contrary, an automatic retrieval hypothesis will predict undistinguishable sequence effects for four kinds of cue sequence conditions.

\section{Experiment 1}

\subsection{Participants}

A total of 33 students (with a mean age of 23 years, range 19 to 27 years, 13 females) consented to participate in this experiment. All participants were right-handed and reported normal or corrected-to-normal vision. All participants were naive as to the purpose of the experiment. 


\subsection{Apparatus and Stimuli}

The stimuli were presented on a LCD display operating at a $75 \mathrm{~Hz}$ frame rate. The participants were seated approximately $60 \mathrm{~cm}$ away from the screen in a dimly-lit room.

A cross, subtending $1^{\circ}$, was placed at the center of the screen as a fixation point. Face photographs were included as central cues. The central stimulus was a photograph of a female face, about $4^{\circ}$ wide and $7^{\circ}$ height, displayed in eight-bit grayscale. The face photograph was manipulated to produce the left-gaze and right-gaze cues by cutting out the pupil/iris area of each eye and pasting it into the left and right corner of each eye, respectively, using Photoshop CS2 software. The target stimulus was a capital letter (' $\mathrm{X}$ ' or ' $\mathrm{O}$ ') measuring $1^{\circ}$ wide, $1^{\circ}$ high, and was presented $15^{\circ}$ away from the fixation point on the left or right side.

\subsection{Design and Procedure}

The cue-target SOAs were $100 \mathrm{~ms}$ and $600 \mathrm{~ms}$. On each trial, cue direction, target location, and SOA duration were selected randomly and equally. Therefore, the cue validity was $50 \%$. For each participant, there were six blocks with 80 trials each. Including 20 training trials, there were in total 500 trials for each participant.

Participants were instructed to keep fixating on the center of the screen. First, a fixation cross appeared at the center of the screen for $1000 \mathrm{~ms}$, and then the cue stimulus appeared. After a certain cue-target SOA, a target letter ' $\mathrm{X}$ ' or ' $\mathrm{O}$ ' appeared either at left or right until participants had responded or $1500 \mathrm{~ms}$ had elapsed. The cue stimulus was still remained on the screen after the appearance of the target. Participants were instructed to respond to the identity of the target letters by pressing the 'UP' key with their middle finger of the right hand or the 'DOWN' key with their index finger of the right hand, the mapping between the responses and the target letters were counter-balanced across the participants. Participants were also informed that the central stimuli did not predict the location in which target would appear, and that they should try to ignore the central cues.

\subsection{Results}

The participants missed or responded to a wrong target at about $2.6 \%$ of the all trials. Anticipations (RT of less than $100 \mathrm{~ms}$ ) and outliers (RT over $1000 \mathrm{~ms}$ ) were classified as errors and were excluded from analysis. After that, responses with RTs exceeding plus or minus two standard deviations of each participant's mean RT on each single cell of the design were also removed as errors. As a result, about $8.4 \%$ of all trials were removed as errors. The error rates did not vary systematically and no signs of any speedaccuracy trade-off were observed. 

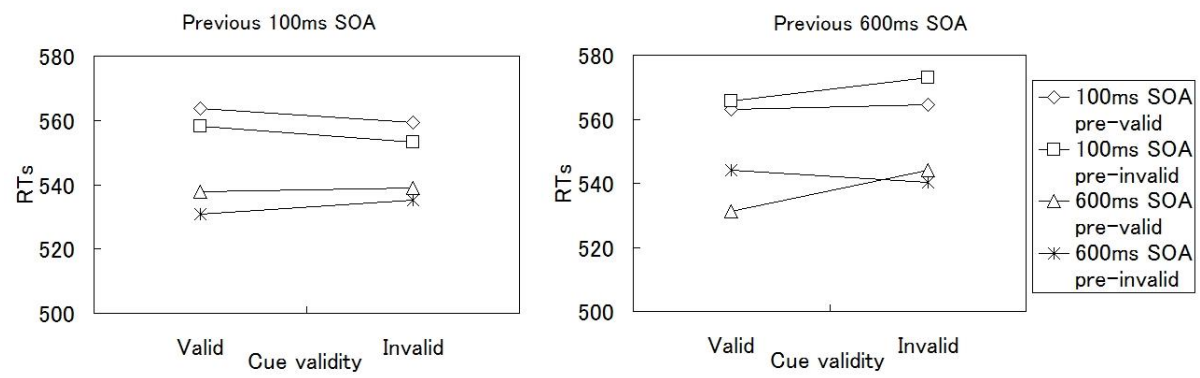

Fig. 1. Mean reaction times (RTs) under different previous and current cue validities and SOAs in Experiment 1.

The mean RTs under different conditions can be seen in Fig. 1. A four-way ANOVA with previous SOA (pre-100 ms and pre-600 ms), SOA (100 ms and $500 \mathrm{~ms}$ ), previous cue validity (pre-valid and pre-invalid), and cue validity (valid and invalid) as within-participants factors was conducted on the RTs. There was a significant main effect of previous SOA, $\mathrm{F}(1,32)=9.122$, $\mathrm{p}<.005$, with RTs becoming slower as the previous SOA was increased. The main effect of SOA was also significant, $\mathrm{F}(1,32)=$ $30.589, \mathrm{p}<.0001$, with RTs becoming shorter as the current SOA was increased. The interaction between previous SOA and previous cue validity was significant, $\mathrm{F}(1,32)$ $=9.096, \mathrm{p}<.005$. This interaction was qualified by a significant previous SOA $\times$ current $\mathrm{SOA} \times$ previous cue validity $\times$ cue validity interaction, $\mathrm{F}(1,32)=4.200, \mathrm{p}<.049$. Further tests revealed that the previous cue validity $\times$ cue validity interaction (indicating the sequence effects) were only significant when both the previous and current SOAs were $600 \mathrm{~ms}, \mathrm{~F}(1,32)=6.787, \mathrm{p}<.014$, but not for other combinations of previous and current SOAs (ps $>.4$ ). The average sequence effects (calculated by cueing effects of pre-valid trials minus cueing effects of pre-invalid trials) for different SOA conditions are $0.3 \mathrm{~ms}$ (pre100-100), -3.2 ms (pre100-600), -6.1 ms (pre600-100), and $16.7 \mathrm{~ms}$ (pre600-600). No other effects or interactions reached significance.

In order to investigate the influence of target locations and target identities on the sequence effects, a four-way ANOVA with target location (repeated and switched), target identity (repeated and switched), previous cue validity (pre-valid and pre-invalid), and cue validity (valid and invalid) as within-participants factors was conducted on the RTs. The main effects of target location and target identity were both significant, $\mathrm{F}(1,32)=15.044, \mathrm{p}<.0001$, and $\mathrm{F}(1,32)=37.151, \mathrm{p}<.0001$, respectively. The interaction between target location and target identity was also significant, $F(1,32)=$ $200.270, p<.0001$, reflecting that alternation of target identity between trials slowed RTs only when target location repeats between trials. The interaction between target location and previous cue validity was significant, $F(1,32)=12.734, p<.001$. No other effects or interactions reached significance.

The results of experiment 1 showed that significant sequence effects can be found in a discrimination task with gaze cues. Furthermore, similar to the finding of previous study [7], sequence effects were only significant under relatively long SOA conditions. However, contrary to the previous study [7], no significant influence of target location 
(or target identity) on the sequence effect was found. This is probably because that the sequence effects are not significant in most of trials when previous or current SOAs are short. An investigation that only involves the pre600-600 SOA condition will be infeasible for the small trial numbers. Therefore, the influence of target location and target identity will be investigated again in experiment 2 .

\section{Experiment 2}

\subsection{Participants}

A total of 36 students (with a mean age of 23 years, range 19 to 27 years, 15 females) consented to participate in this experiment. All participants were right-handed and reported normal or corrected-to-normal vision. All participants were naive as to the purpose of the experiment.

\subsection{Apparatus and Stimuli}

The apparatus and the stimuli were the same as that in experiment 1 except that an arrow cue was included. For arrow cues, the central fixation stimulus was a horizontal line centered on the screen, $3^{\circ}$ in length. An arrow head and an arrow tail appeared at the ends of the central line, both pointing left or both pointing right. The length of an arrow, from the tip of the arrow head to the ends of the tail, was $4^{\circ}$.

\subsection{Design and Procedure}

A relatively long SOA (600 ms) was used to ensure the capability of the design to induce sequence effects. On each trial, cue type (gaze cue or arrow cue), cue direction, and target location were selected randomly and equally. There were eight blocks with 80 trials each. Including 20 training trials, there were in total 660 trials for each participant. The procedure was the same as that in experiment 1 .

\subsection{Results}

The participants missed or responded to wrong targets at an average of about $2.1 \%$ of all trials. Anticipations (RT of less than $100 \mathrm{~ms}$ ) and outliers (RT over $1000 \mathrm{~ms}$ ) were classified as errors and were excluded from analysis. After that, responses with RTs exceeding plus or minus two standard deviations of the participant's mean RT on each single cell of the design were also removed as errors. As a result, about $7.5 \%$ of all trials were removed as errors. The error rates did not vary systematically and no signs of any speed-accuracy trade-off were observed.

A four-way ANOVA with previous cue type (pre-gaze or pre-arrow), cue type (gaze or arrow), previous cue validity (pre-valid and pre-invalid), and cue validity (valid and invalid) as within-participants factors was conducted on the RTs. There was a significant main effect of cue validity, $\mathrm{F}(1,35)=50.446, \mathrm{p}<.0001$, demonstrating the cueing 
effect, and a significant interaction between previous validity and cue validity, $\mathrm{F}(1,35)$ $=14.06, \mathrm{p}<.001$, indicating the significant sequence effect. As for the influence of cue types, the interaction between previous cue type and previous cue validity was significant, $\mathrm{F}(1,35)=8.265, \mathrm{p}<.007$, reflecting that RTs were shorter for pre-valid than for pre-invalid condition when the previous cue was an arrow, but this tendency was reversed when the previous cue was a gaze. The interaction between previous cue type and cue validity was also significant, $\mathrm{F}(1,35)=4.548, \mathrm{p}<.04$, indicating that cueing effects were larger for pre-arrow condition than for pre-gaze condition. No other factors or interactions were significant. The average sequence effects for different cue sequence conditions are $11.1 \mathrm{~ms}$ (arrow-face sequence), $10.6 \mathrm{~ms}$ (arrow-arrow), $7.1 \mathrm{~ms}$ (face-arrow), and $7.2 \mathrm{~ms}$ (face-face). Paired-samples t tests confirmed that the magnitude of these four sequence effects is not significantly different from each other (ps > .59). The mean RTs under different cue sequence conditions can be seen in Fig. 2.
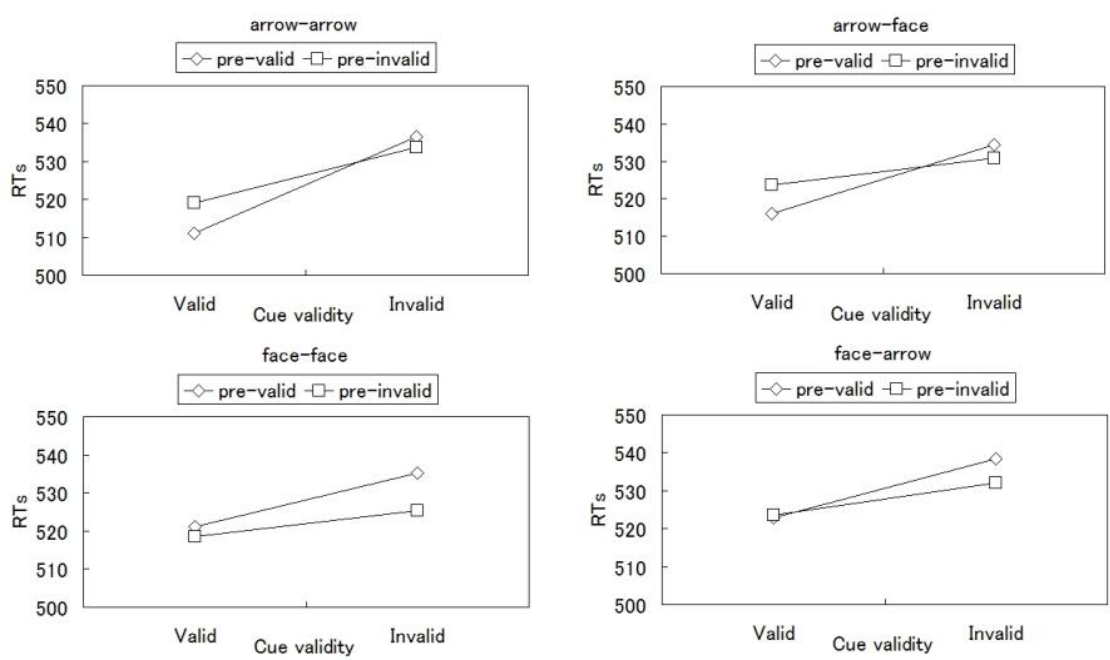

Fig. 2. Mean reaction times (RTs) under different previous and current cue validities and different combination of cue types between trials in Experiment 2.

Similar to the experiment 1, a four-way ANOVA with target location (repeated and switched), target identity (repeated and switched), previous cue validity (pre-valid and pre-invalid), and cue validity (valid and invalid) as within-participants factors was conducted on the RTs. The main effects of target location and target identity were both significant, $\mathrm{F}(1,35)=31.779, \mathrm{p}<.0001$, and $\mathrm{F}(1,35)=35.923$, $\mathrm{p}<.0001$, respectively. Similar to that in experiment 1 , the interaction between target location and target identity was significant, $\mathrm{F}(1,35)=121.552, \mathrm{p}<.0001$, demonstrating that alternation of target identity between trials slowed RTs only when target location repeats between trials. The interaction between target location and previous cue validity was also significant, $\mathrm{F}(1,35)=25.232, \mathrm{p}<.0001$. In addition, there was a significant main effect of cue validity, $F(1,35)=41.302, p<.0001$, demonstrating the cueing effect, and a significant interaction between previous validity and cue validity, $F(1,35)=12.774$, $p$ 
$<.001$, indicating the sequence effect. Importantly, the target location $\times$ previous cue validity $\times$ cue validity interaction were marginally significant, $F(1,35)=3.989, p$ $=.054$, replicating the influence of target locations on the sequence effect that was observed in the previous study. No other effects or interactions reached significance.

In all, significant sequence effects were found for all four combination conditions of two cue types. In addition, although the influence of target locations on the sequence effect was replicated, no significant influence of target identity alternation was found.

\section{Discussion}

The present study investigated the sequence effects (i.e., trial-by-trial influence) induced by gaze and arrow cues in cueing paradigm. In experiment 1 , participants were asked to discriminate targets in a gaze cueing task, and it was found that gaze cues induced significant sequence effects in long SOA conditions. In addition, sequence effects were not influenced by the repetition and switch of target identities and its corresponding responses. When gaze and arrow cues were intermixed in experiment 2 , the four combination conditions of cue types induced significant and similar sequence effects. In addition, though the influence of target locations on the sequence effects that was reported previously [7] was replicated, no significant influence of target identities was found.

Results from experiment 1 extend our knowledge about the sequence effects for the following aspects. First, sequence effects still exist when gazes are used as central cues. This finding suggests that sequential processing is a common phenomenon in symbolic cueing paradigm and is not limited to specific cue types, such as arrows.

Second, the appearance of sequence effects does not depend on specific task demands. So far as we know, all previous studies used the simple detection task to test the sequence effects. Before this study, it remains unknown whether or not the sequence effects can be found with different tasks.

Third, both experiment 1 and experiment 2 found that overall RTs were slowed down by the alternation of target identities (at least when target location repeated between trials), probably because participants need time to choose a different valid response key for a changed target. However, sequence effects were not influenced by the repetition and switch of target identities (along with its responses). From a memory perspective, there may be two major phases for the sequence processes: initial encoding phase in previous trials and later retrieval phase in current trials. In the former phase, the spatial organization information between cue directions and target locations needs to be encoded into memory; in the later phase, the information will be retrieved from memory to affect performance. Current results may reflect some memory mechanisms during the sequential processing. That is, the target identity information is not encoded and retrieved between trials.

Sequence effects by gaze and arrow cues were directly compared in experiment 2. Gaze and arrow cues were intermixed in a within-block design. Such design resulted four kinds of cue sequence conditions between consecutive trials: gaze-gaze sequence, 
gaze-arrow sequence, arrow-gaze sequence, arrow-arrow sequence. Interestingly, sequence effects are significant for all sequence conditions despite the alternation of cue types. That means gaze cues induce the same amount of sequence effects as arrow cues, and the sequence effect can generalize across different cue types (from gaze to arrow, or from arrow to gaze). This finding supports the automatic retrieval hypothesis and suggests that sequence effects induced by gaze and arrow cues are processed in the same system, probably the so-called implicit memory system in human brain $[9,10]$.

In all, the present study investigated the sequence effect in gaze cueing paradigm. Similar to the previous findings from arrow cueing, significant sequence effects were found. The results extend our knowledge by showing that sequence effects in cueing paradigm are not limited to a specific cue type or a specific task, and suggest that sequential processing is a common mechanism in attention orienting systems.

Acknowledgments. This research is supported by the NSFC (31300938, 61462053, and 61403251), and the Yunnan NSFC(2016FB107).

\section{References}

1. Posner, M.: Orienting of attention. Quarterly Journal of Experimental Psychology. 32, 3--25 (1980)

2. Hommel, B., Pratt, J., Colzato, L., Godijn, R.: Symbolic control of visual attention. Psychological Science. 12, 360--365 (2001)

3. Frischen, A., Bayliss, A., Tipper, S.: Gaze cueing of attention: Visual attention, social cognition, and individual differences. Psychological Bulletin. 133, 694--724 (2007)

4. Jongen, E., Smulders, F.: Sequence effects in a spatial cueing task: Endogenous orienting is sensitive to orienting in the preceding trial. Psychological Research. 71, 516--523 (2007)

5. Gómez, C.M., Flores, A., Digiacomo, M.R., Vázquez-Marrufo, M.: Sequential P3 effects in a Posner's spatial cueing paradigm: trial-by-trial learning of the predictive value of the cue. Acta Neurobiol. Exp. 69, 155--167 (2009)

6. Dodd, M., Pratt, J.: The effect of previous trial type on inhibition of return. Psychological Research. 71, 411--417 (2007)

7. Qian, Q., Shinomori, K., Song, M.: Sequence effects by non-predictive arrow cues. Psychological Research. 76, 253--262 (2012)

8. Qian Q., Song, M., Shinomori, K.: Gaze cueing as a function of perceived gaze direction. Japanese Psychological Research. 55, 264--272 (2013)

9. Maljkovic, V., Nakayama, K.: Priming of pop-out: III. A short-term implicit memory system beneficial for rapid target selection. Visual Cognition. 7, 571--595 (2000) 
10. Kristjánsson, A.: Rapid learning in attention shifts: A review. Visual Cognition. 13, 324--362 (2006) 